\title{
A closer look at the cosmological implications of the $\Lambda$ HDE model
}

\author{
Shuang Wang ${ }^{1 \star}$ Sixiang Wen ${ }^{1}$, Lanjun Zhou ${ }^{2,3}$, Miao Li $^{1}$ \\ ${ }^{1}$ School of Physics and Astronomy, Sun Yat-Sen University, Zhuhai 519082, P. R. China \\ ${ }^{2}$ Key Laboratory of Theoretical Physics, Institute of Theoretical Physics, Chinese Academy of Sciences, Beijing 100190, P. R. China \\ ${ }^{3}$ School of Physical Science, University of Chinese Academy of Science, Beijing 100049, P. R. China
}

11 July 2018

\begin{abstract}
In a previous paper $\mathrm{Hu}$ et al. (2015), we proposed a heterotic dark energy model, called $\Lambda \mathrm{HDE}$, in which dark energy is composed of two components: cosmological constant (CC) and holographic dark energy (HDE). The aim of this work is to give a more comprehensive and systematic investigation on the cosmological implications of the $\Lambda$ HDE model. Firstly, we make use of the current observations to constrain the $\Lambda$ HDE model, and compare its cosmology-fit results with the results of the $\Lambda \mathrm{CDM}$ and the HDE model. Then, by combining a qualitative theoretical analysis with a quantitative numerical study, we discuss the impact of considering curvature on the cosmic evolutions of fractional HDE density $\Omega_{h d e}$ and fractional CC density $\Omega_{\Lambda}$, as well as on the ultimate cosmic fate. Finally, we explore the effects of adopting different types of observational data. We find that: (1) the current observational data cannot distinguish the $\Lambda \mathrm{HDE}$ model from the $\Lambda \mathrm{CDM}$ and the HDE model; this indicates that DE may contain multiple components. (2) the asymptotic solution of $\Omega_{h d e}$ and the corresponding cosmic fate in a flat universe can be extended to the case of a non-flat universe; moreover, compared with the case of a flat universe, considering curvature will make HDE closer to a phantom dark energy. (3) compared with JLA dataset, SNLS3 data more favor a phantom type HDE; in contrast, using other types of observational data have no significant impact on the cosmic evolutions of the $\Lambda \mathrm{HDE}$ model.
\end{abstract}

Key words: cosmology: dark energy, observations, cosmological parameters

\section{INTRODUCTION}

Since its discovery in 1998 Riess et al. (1998); Perlmutter et al. (1999), dark energy (DE) has become one of the central problems in modern cosmology Frieman, Turner \& Huterer (2008); Caldwell \& Kamionkowski (2009); Li et al. (2011 2013c); Wang, Wang \& Li (2016). Although numerous DE models Steinhardt, Wang \& Zlatev (1999); Zlatev, Wang \& Steinhardt (1999); Armendariz, Damour \& Mukhanov (1999); Kamenshchik, Moschella \& Pasquier (2001); Bento, Bertolami \& Sen (2002); Caldwell (2002); Malquarti et al. (2003); Wei, Cai \& Zeng (2005); Cai (2007); Zhang, Xia \& Zhao (2007); Wang \& Zhang (2008); Wang, Zhang, \& Xia (2008); Wei \& Cai (2009); Gao, Chen \& Shen (2009); Huang et al. (2009); Wang, Li \& Li (2011) have been proposed, the nature of DE is still a mystery.

In principle, the DE problem should be an issue of quantum gravity Witten (2000). It is commonly believed that the holographic principle' 't Hooft (1993); Susskind (1995) is a fundamental principle of quantum gravity. Based on the holographic princi-

\footnotetext{
^ wangshuang@mail.sysu.edu.cn
}

ple, one of the present authors (ML) proposed the holographic dark energy (HDE) model Li (2004). In this model, the HDE density $\rho_{\text {hde }}$ can be described by

$$
\rho_{\text {hde }}=3 C^{2} M_{p}^{2} L^{-2},
$$

where $C$ is a key parameter to label the magnitude of holographic contribution to DE, $M_{p}^{2}=(8 \pi G)^{-1}$ is the reduced Planck mass, and $G$ is Newtonian gravitational constant. Li suggested to choose the future event horizon $\left(R_{h}=a \int_{t}^{+\infty} \frac{d t}{a}\right)$ as the cutoff $L \mathrm{Li}$ (2004) 1 . This model is the first DE model inspired by the holographic principle, and it is in good agreement with the current cosmological observations Huang \& Gong (2004); Shen et al. (2005);

\footnotetext{
1 The original HDE model have the circular logic problem. In other words, the existence of the future event horizon needs the accelerating expansion, while the dark energy component that cause accelerating expansion is given by the future event horizon. Due to this problem, the original HDE model cannot be derived by the action principle. But in a paper of our group $\mathrm{Li} \&$ Miao 2012), we have constructed a action, which can derive the energy density form of HDE from the first principle. This means that, although it seems that the original HDE model has the circular logic problem, this problem is not a real problem in the framework of HDE.
} 
Chang, Wu \& Zhang (2006); Zhang \& Wu. (2007); Li et al. (2009a b); Zhang et al. (2012); Li et al. (2013b ? a). In recent years, the HDE model has attracted lots of interests Horvat (2004); Wang, Gong \& Abdalla (2005); Pavon \& Zimdahl (2005); Nojiri \& Odintsov (2006); Setare (2006, 2007); Saridakis (2007). Our group has also done a series of research works about HDE. For examples, in Li, Lin \& Wang (2008), the HDE model was proved to be a perturbatively stable model; in Li et al. (2009a), it was found that the original HDE model is more favored by the current observations than other types of holographic DE models Cai (2007); Gao, Chen \& Shen (2009); in Li, Miao \& Pang (2010), it was proved that the Casimir energy of the photon field in a de Sitter space have the same form of HDE; in Li \& Wang (2010), the entropic force formalism was applied to cosmology, which cause that the HDE appear in the Friedmann equation.

In almost all the DE models, DE contains only a single component. However, since baryonic matter contains multiple components, while dark matter may also contain multiple components Bertone, Hooper \& Silk (2005), it will be very interesting to take into account the possibility that DE contains multiple components. In the recent paper $\mathrm{Hu}$ et al. (2015), we proposed the $\Lambda$ HDE model 2 which contains two DE components: cosmological constant (CC) and HDE. So far as we know, this is the first theoretical attempt to explore the possibility that DE may contain multiple components. In $\mathrm{Hu}$ et al. (2015), we simply discussed the cosmic evolutions of the $\Lambda \mathrm{HDE}$ model in a flat universe, and constrain this model with some observational data. However, it must be pointed out that there are still some shortcomings for Ref. Hu et al. (2015): (1) the cosmology-fit results of the $\Lambda$ HDE model have not been compared with the results of other DE models; (2) the impact of curvature on the cosmic evolutions and the cosmic fates of the $\Lambda$ HDE model have not been considered; (3) the effects of adopting different types of observational data have not been taken into account.

In this work, we will make a more comprehensive and systematic investigation on the $\Lambda$ HDE model. Firstly, we make use of a combination of the type Ia supernovae (SNe Ia), the Baryon Acoustic Oscillations (BAO), the Cosmic Microwave Background (CMB) and the Growth Fact (GF) data to constrain the $\Lambda \mathrm{HDE}$ model, and then compare its cosmology-fit results with the results of the $\Lambda$ cold-dark-matter $(\Lambda \mathrm{CDM})$ model and the HDE model. Secondly, we extend the discussions about the cosmic evolution of $\Omega_{h d e}$ in a flat universe to the case of a non-flat universe. We not only perform a qualitatively analysis on the evolution of $\Omega_{h d e}$, but also give a quantitative result by using the current cosmological observations. Thirdly, in order to explore the impact of different datasets on the evolutions of DE, we adopt two SN datasets, two BAO datasets and two CMB datasets; in addition, we also study the impact of adding growth factor data or not.

Different from Ref. Hu et al. (2015), in this work we study a new version of $\Lambda$ HDE model: in the deceleration expansion stage, $\mathrm{DE}$ only contains the $\mathrm{CC}$ term; in the accelerated expansion stage, DE contains both the $\mathrm{CC}$ and the HDE. We describe our method in section 2 present our results in section 3 , and summarize in section 4 In this paper, we assume today's scale factor $a_{0}=1$, thus the

2 There is an implicit assumption in the $\Lambda$ HDE model that once the accelerated expansion commences it will never end. It cannot be excluded that the quantum vacuum has the possibility of decaying into radiation and/or matter particles Freese et al. (1987); Polyakov 2010). However, in this work, we assume that the vacuum will not decay. redshift $z=a^{-1}-1$. The subscript " 0 " always indicates the present value of the corresponding quantity.

\section{METHODOLOGY}

In this section, firstly we review the theoretical framework of the $\Lambda \mathrm{HDE}$ model, then we briefly describe the observational data used in the present work.

\subsection{The $\Lambda$ HDE model}

In a non-flat universe, the Friedmann equation can be written as

$$
3 M_{p}^{2} H^{2}=\rho_{m}+\rho_{d e}+\rho_{r}+\rho_{k},
$$

where $H \equiv \dot{a} / a$ is the Hubble parameter (the dot denotes the derivative with respect to time $t), \rho_{m}, \rho_{d e}, \rho_{k}$ and $\rho_{r}$ are the energy densities of matter, DE, curvature and radiation, respectively. Note that $\rho_{m}$ is the sum of baryon density $\rho_{b}$ and dark matter density $\rho_{d m}$. For convenience, we define the fractional energy density of each component as:

$$
\Omega_{x} \equiv \frac{\rho_{x}}{\rho_{c}}, \quad \rho_{c}=\rho_{m}+\rho_{d e}+\rho_{r}+\rho_{k},
$$

where the subscript $x$ can represent arbitrary cosmological component.

As mentioned above, in this work we study a modified version of $\Lambda \mathrm{HDE}$ model: in the deceleration expansion stage, DE only contains the $\mathrm{CC}$ term; in the accelerated expansion stage, DE contains both the CC and the HDE. The latest Planck 2015 paper Ade et al. (2015a) gives a best-fit value $\Omega_{m 0}=0.308$; this implies that the expansion of our universe begin to accelerate at $z \simeq 0.65$. Thus the total energy density can be written as

$\rho_{d e}= \begin{cases}\rho_{\Lambda}, & z>0.65 \\ \rho_{\Lambda}+\rho_{\text {hde }}, & z \leqslant 0.65\end{cases}$

Here $\rho_{\Lambda}=M_{p}^{2} \Lambda$ and $\rho_{h d e}=3 C^{2} M_{p}^{2} / L^{2}$ are the energy density of CC and HDE, respectively. The cutoff length scale $L$ takes the form Huang \& Li (2004)

$$
L=\frac{a}{\sqrt{\left|\Omega_{k 0}\right|} H_{0}} \operatorname{sinn}\left(\sqrt{\left|\Omega_{k 0}\right|} H_{0} \int_{t}^{+\infty} \frac{d t^{\prime}}{a}\right),
$$

where the function $\operatorname{sinn}(x)$ is defined as

$\operatorname{sinn}(x)= \begin{cases}\sin (x), & \text { if } \Omega_{k 0}<0 \\ x, & \text { if } \Omega_{k 0}=0 \\ \sinh (x), & \text { if } \Omega_{k 0}>0\end{cases}$

Let us focus on the cosmic evolution of the $\Lambda$ HDE model at the redshift region $z \leqslant 0.65$. Following the procedure of $\mathrm{Hu}$ et al. 【2015), we can get the following differential equations for the reduced Hubble parameter $E(z) \equiv H(z) / H_{0}$ and the fractional HDE density $\Omega_{h d e}(z)$ :

$$
\begin{aligned}
\frac{1}{E(z)} \frac{d E(z)}{d z}= & -\frac{\Omega_{h d e}}{1+z}\left(\frac{3 \Omega_{\Lambda}+\Omega_{k}-\Omega_{r}-3}{2 \Omega_{h d e}}+\frac{1}{2}+\right. \\
& \left.+\sqrt{\frac{\Omega_{h d e}}{C^{2}}+\Omega_{k}}\right) \\
\frac{d \Omega_{h d e}(z)}{d z}= & -\frac{2 \Omega_{h d e}\left(1-\Omega_{h d e}\right)}{1+z}\left(\sqrt{\frac{\Omega_{h d e}}{C^{2}}+\Omega_{k}}+\frac{1}{2}-\right. \\
& \left.-\frac{3 \Omega_{\Lambda}+\Omega_{k}-\Omega_{r}}{2\left(1-\Omega_{h d e}\right)}\right) .
\end{aligned}
$$


Making use of the initial condition $E(0)=1$ and $\Omega_{h d e}(z=0)=$ $\Omega_{h d e 0}$, the Eqs. 7 and 8 can be solved numerically.

\subsection{Observational Data}

We will make use of the following datasets to constrain the $\Lambda \mathrm{HDE}$ model.

- The SNLS3 combined sample (hereafter SNLS3) consists of $472 \mathrm{SNe}$ Ia Conley et al. (2011). This sample has been widely used in the studies of cosmology Li et al. (2011 2012); Wang et al. (2016). Its $\chi^{2}$ function can be written as:

$$
\chi_{S N L S 3}^{2}=\Delta \mathbf{m}^{T} \cdot \mathbf{C o v}^{-1} \cdot \Delta \mathbf{m},
$$

where Cov is the total covariance matrix, which is given by Conley et al. (2011), and $\Delta \mathbf{m} \equiv \mathbf{m}_{B}-\mathbf{m}_{\bmod }$ is a data vector. Here $\mathbf{m}_{B}$ is the observed peak magnitude in rest-frame $B$ band, $\mathbf{m}_{m o d}$ is predicted magnitude of $\mathrm{SNe}$ Ia, given by

$$
\mathbf{m}_{\text {mod }}=5 \log _{10}\left[\frac{d_{L}}{M p c}\right]+25-\alpha \times(s-1)+\beta \times \mathcal{C}+M,
$$

where $s$ describes the time stretching of the light-curve, $\mathcal{C}$ describes the supernova color at maximum brightness, $M$ is the absolute magnitude, and $d_{L}$ is the luminosity distance (the corresponding expression is given in Wang \& Wang (2013a)). Notice that the stretch-luminosity parameter $\alpha$ and the color-luminosity parameter $\beta$ are free parameters ${ }^{3}$

For comparison, we also use the JLA supernova samples (hereafter JLA) Betoule et al. (2014).

- The BAO data we used is extracted from three BAO measurements: the Baryon Oscillation Spectroscopic Survey Data Release 9(BOSS DR9) Eisenstein et al. (2011), the Sloan Digital Sky Survey Data Release 7(SDSS DR7) Abazajian et al. (2009) and the 6dF Galaxy Survey (6dFGS) Beutler et al. (2011). There are two kinds of BAO data: the first is extracted by using the spherically averaged one-dimensional galaxy clustering statistics (hereafter "BAO1d"), while the second is obtained by using the anisotropic two-dimensional GC statistics (hereafter "BAO2d") Wang, Hu \& Li (2015).

BAO1d includes the measurement of $r_{s}\left(z_{d}\right) / D_{v}(z=0.106)$ from Beutler et al. (2011), $D_{v}(z=0.35) / r_{s}\left(z_{d}\right)$ from Abazajian

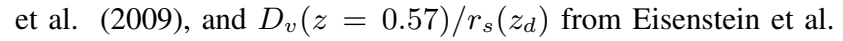
(2011). Here $r_{s}\left(z_{d}\right)$ is the comoving sound horizon at "drag" epoch and $D_{v}(z)$ is a volume averaged distance indicator (the corresponding expressions are given in Wang (2014)). For convenience, we define:

$$
\begin{aligned}
\mathbf{q} \equiv & \left(r_{s}\left(z_{d}\right) / D_{v}(z=0.106), D_{v}(z=0.35) / r_{s}\left(z_{d}\right),\right. \\
& \left.D_{v}(z=0.35) / r_{s}\left(z_{d}\right)\right) .
\end{aligned}
$$

\footnotetext{
${ }^{3}$ It should be mentioned that, previous studies on the SNLS3 dataset Wang \& Wang (2013a) and JLA dataset Li et al. (2016) found strong evidence for the redshift-dependence of color-luminosity parameter $\beta$, and this conclusion has significant effects on parameter estimation of various cosmological models Wang, Li \& Zhang (2014); Wang et al. (2014); Wang, Wang \& Zhang (2014); Wang et al. (2015); Wang, Wen \& Li (2016). In addition, different light-curve fitters of SNIa can also affect the results of cosmologyfits Bengochea 2011; Mohlabeng \& Ralston (2013); Hu et al. (2016). But in this work, for simplicity, we just adopt the most mainstream recipe of processing SNLS3 data, and do not consider the factors of time-varying $\beta$ and different light-curve fitters.
}

Then we can represent the $\chi^{2}$ function of BAO1d dataset as

$$
\chi_{B A O 1 d}^{2}=\sum_{i} \frac{\left(q_{i}-q_{i}^{\text {data }}\right)^{2}}{\sigma\left(q_{i}\right)^{2}},
$$

where $q_{i}^{d a t a}$ and $\sigma\left(q_{i}\right)$ are the observed value and the $1 \sigma$ error of $q_{i}$.

BAO2d includes the measurement of $r_{s}\left(z_{d}\right) / D_{v}(z=0.106)$ from Beutler et al. (2011), the measurements of $H(z=$ $0.35) r_{s}\left(z_{d}\right) / c$ and $D_{A}(z=0.35) / r_{s}\left(z_{d}\right)$ from (Hemantha, Wang \& Chuang 2014), as well as the measurements of $H(z=$ $0.57) r_{s}\left(z_{d}\right) / c$ and $D_{A}(z=0.57) / r_{s}\left(z_{d}\right)$ from Wang (2014). For convenience, we define:

$$
\begin{aligned}
\mathbf{p} \equiv & \left(r_{s}\left(z_{d}\right) / D_{v}(z=0.106), H(z=0.35) r_{s}\left(z_{d}\right) / c\right. \\
& D_{A}(z=0.35) / r_{s}\left(z_{d}\right), H(z=0.57) r_{s}\left(z_{d}\right) / c \\
& \left.D_{A}(z=0.57) / r_{s}\left(z_{d}\right)\right)
\end{aligned}
$$

Then we can represent the $\chi^{2}$ function of BAO2d dataset as

$$
\chi_{B A O 2 d}^{2}=\sum_{i, j}\left(p_{i}-p_{i}^{d a t a}\right)\left(\operatorname{Cov}_{B A O 2 d}^{-1}\right)_{i j}\left(p_{j}-p_{j}^{\text {data }}\right),
$$

where $p_{i}^{\text {data }}$ is the observed value of $p_{i}$ and $\operatorname{Cov}_{B A O 2 d}$ is the covariance matrix. For the details of the covariance matrix $\operatorname{Cov}_{B A O 2 d}$, we refer the reader to the Refs. Hemantha, Wang \& Chuang (2014); Wang (2014).

- The cosmic microwave background data we used is the distance priors data extracted from Planck 2015 data release (hereafter Planck 2015) Ade et al. (2015b) This dataset use three quantities $l_{a}, R$ and $\Omega_{b 0} h^{2}\left(h \equiv H_{0} / 100\right)$ to provide an efficient summary of CMB data. Here $l_{a}, R$ and $\Omega_{b 0} h^{2}$ are the acoustic scale, the CMB shift parameter and the present baryon component, respectively. All these quantities are defined as follows:

$$
\begin{aligned}
l_{a} & =\pi r\left(z_{*}\right) / r_{s}\left(z_{*}\right) \\
R & =\sqrt{\Omega_{m 0} H_{0}^{2}} r\left(z_{*}\right) / c \\
\Omega_{b 0} & =\rho_{b 0} /\left(3 M_{p}^{2} H_{0}^{2}\right)
\end{aligned}
$$

where $z_{*}$ is the redshift to the photon-decoupling surface given in Ref. (Hu \& Sugiyama 1996), $r\left(z_{*}\right)$ is the comoving distance to $z_{*}$, and $r_{s}\left(z_{*}\right)$ is the comoving sound horizon at $z_{*}$ (the corresponding expressions are given in Wang \& Wang (2013b)). For convenience, let's define: $\mathbf{p a r} \equiv\left(l_{a}, R, \Omega_{b 0} h^{2}\right)$. Then the $\chi^{2}$ function can be written as

$$
\chi_{C M B}^{2}=\sum_{i, j}\left(\operatorname{par}_{i}-\operatorname{par}_{i}^{d a t a}\right)\left(\operatorname{Cov}_{C M B}^{-1}\right)_{i j}\left(\operatorname{par}_{j}-\operatorname{par}_{j}^{\text {data }}\right),
$$

where $\operatorname{par}_{i}^{\text {data }}$ is the observed value of $\operatorname{par}_{i}$ and $\operatorname{Cov}_{C M B}$ is the covariance matrix, which is given in the Refs. Ade et al. (2015b).

For comparison, we also use the distance priors data extracted from Planck 2013 data release (hereafter Planck 2013) Wang \& Wang (2013b).

- The linear perturbation theory tell us

$$
\ddot{\delta_{m}}+2 H \dot{\delta_{m}}-4 \pi G \rho_{m} \delta_{m}=0,
$$

where $\delta_{m} \equiv \delta \rho_{m} / \rho_{m}$ is the matter density perturbation. Assuming that $D(z)$ is a solution of Eq. 19 it is clear that $D(0)=1$ and $D(\infty)=0$. Therefore, the growth rate of large scale structure

\footnotetext{
4 In addition to Ade et al. 2015b, there are some other distance priors data extracted from the Planck 2015 data release, i.e. see Refs Wang \& Dai |2016); Huang, Wang \& Wang 2015); Wang \& Wang (2013b).
} 
is $f(z)=-d \ln D / d \ln (1+z)$, and the root-mean-square mass fluctuation in $8 h^{-1} \mathrm{Mpc}$ spheres is $\sigma_{8}(z)=\sigma_{8}^{0} D(z)$, where $\sigma_{8}^{0}$ is the current value of $\sigma_{8}(z)$. So we can get Pavlov, Farooq \& Ratra (2014)

$$
\chi_{g}^{2}\left(\sigma_{8}^{0}\right)=\sum_{i=1}^{N} \frac{\left.A\left(z_{i}, \sigma_{8}^{0}\right)-A_{\text {data }}\left(z_{i}\right)\right]^{2}}{\sigma_{i}^{2}},
$$

where $N$ is the number of data points, $A\left(z, \sigma_{8}^{0}\right) \equiv f(z) \sigma_{8}(z)$ is the growth parameter, $A_{d a t a}\left(z_{i}\right)$ and $\sigma_{i}$ are the mean value and $1 \sigma$ error of $A$. All the GF data can be obtained from the first table of (Pavlov, Farooq \& Ratra 2014). Moreover, the posterior probability density function $\mathcal{L}_{g}$ is given by Pavlov, Farooq \& Ratra (2014)

$$
\mathcal{L}_{g}=\frac{1}{\sigma_{\overline{\sigma_{8}^{0}}} \sqrt{2 \pi}} \int_{0}^{\infty} \exp \left\{-\frac{\chi_{g}^{2}\left(\sigma_{8}^{0}\right)}{2}-\frac{\left[\sigma_{8}^{0}-\overline{\sigma_{8}^{0}}\right]^{2}}{2 \sigma_{\overline{\sigma_{8}^{0}}}^{2}}\right\} \mathrm{d} \sigma_{8}^{0} .
$$

where $\overline{\sigma_{8}^{0}}=0.813\left(\Omega_{m 0} / 0.25\right)^{-0.47}$ is the mean value of $\sigma_{8}^{0}$, and $\sigma \overline{\sigma_{8}^{0}}=\sqrt{\sigma_{\sigma_{8}^{0}}^{2}+b^{2}}\left(\Omega_{m 0} / 0.25\right)^{-0.47}$ is the $1 \sigma$ uncertainty of $\sigma_{8}^{0}$. The final $\chi^{2}$ function of GF data can be written as

$$
\chi_{g}^{2}=-2 \ln \mathcal{L}_{g} .
$$

In the original paper $\mathrm{Hu}$ et al. (2015), the authors choose

$$
\mathbf{P}=\left\{\Omega_{m 0} h^{2}, \Omega_{b 0} h^{2}, h, C, \Omega_{\Lambda 0}, \Omega_{k 0}, \alpha, \beta\right\}
$$

as a set of free parameters to perform an MCMC likelihood analysis ${ }^{5}$ However, this choice will lead to the result of $\Omega_{h d e 0}$ has a negative $2 \sigma$ lower bound, which is unphysical. So in this work we choose

$$
\mathbf{P}=\left\{\Omega_{b 0}, h, C, \Omega_{h d e 0}, \Omega_{d e 0}, \Omega_{k 0}, \alpha, \beta\right\}
$$

as a set of free parameters. Moreover, we require that $\Omega_{h d e 0}>0$ is always satisfied. Note that $\Omega_{d e 0}=\Omega_{h d e 0}+\Omega_{\Lambda 0}$ is the total fractional DE density of today.

\section{RESULTS}

In this section, First of all, we make use of the SNLS3+BAO1d+Planck 2015+GF data to constrain the $\Lambda \mathrm{HDE}$ model, and compare its cosmology-fit results with the results of the $\Lambda$ CDM and the HDE model. Then, we discuss the impact of curvature on the cosmic evolutions of $\Omega_{\Lambda}$ and $\Omega_{h d e}$ from both the theoretical and the observational aspects. Finally, we explore the effects of adopting different types of observational data on the cosmic evolutions and the cosmic fate.

\subsection{A comparison of the cosmology-fit results of different DE models}

By using the SNLS3+BAO1d+Planck 2015+GF data, we present the cosmology-fit results of the $\Lambda \mathrm{HDE}$ model in the table 1 For comparison, the cosmology-fit results of the $\Lambda \mathrm{CDM}$ model and the HDE model are also listed. We find that the results of $\Omega_{k 0}$ of the three DE models are consistent with the result of a flat universe at $1 \sigma$ confidence level (CL), which are also consistent with the result of Ade et al. (2015a). In addition, the best-fit value of $C$ of the

\footnotetext{
${ }^{5} \Omega_{r 0}$ can be calculated by $\Omega_{r 0}=\Omega_{\gamma 0}\left(1+0.2271 N_{e f f}\right)$, where $\Omega_{\gamma 0}=$ $2.469 \times 10^{-5} h^{-2}$ and $N_{\text {eff }}=3.046$.
}

Table 1. Cosmology-fit results, $\chi_{\text {min }}^{2} / d o f$, AIC and BIC of the $\Lambda$ CDM, the HDE and the $\Lambda$ HDE model. The SNLS3+BAO1d+Planck 2015+GF data are used in the analysis. Both the best-fit values and the $1 \sigma$ errors of various parameters are listed.

\begin{tabular}{llll}
\hline \hline Parameter & $\Lambda \mathrm{CDM}$ & $\mathrm{HDE}$ & $\Lambda \mathrm{HDE}$ \\
\hline$\alpha$ & $1.41_{-0.24}^{+0.30}$ & $1.45_{-0.31}^{+0.29}$ & $1.41_{-0.27}^{+0.35}$ \\
$\beta$ & $3.24_{-0.26}^{+0.29}$ & $3.25_{-0.29}^{+0.32}$ & $3.27_{-0.31}^{+0.29}$ \\
$\Omega_{k 0}$ & $-0.001_{-0.008}^{+0.007}$ & $0.003_{-0.008}^{+0.011}$ & $-0.006_{-0.012}^{+0.013}$ \\
$\Omega_{b 0}$ & $0.048_{-0.004}^{+0.003}$ & $0.046_{-0.005}^{+0.005}$ & $0.047_{-0.005}^{+0.005}$ \\
$h$ & $0.689_{-0.025}^{+0.025}$ & $0.701_{-0.036}^{+0.036}$ & $0.693_{-0.034}^{+0.040}$ \\
$\mathrm{C}$ & & $0.661_{-0.125}^{+0.233}$ & $0.334_{-0.333}^{+2.666}$ \\
$\Omega_{\text {he0 }}$ & & $0.710_{-0.036}^{+0.028}$ & $0.220_{-0.220}^{+0.386}$ \\
$\Omega_{\text {de0 }}$ & $0.704_{-0.023}^{+0.021}$ & $0.710_{-0.036}^{+0.028}$ & $0.713_{-0.033}^{+0.031}$ \\
\hline \hline$\chi_{\text {min }}^{2} /$ dof & 0.912 & 0.914 & 0.913 \\
$\Delta$ AIC & 0 & 1.955 & 2.574 \\
$\Delta$ BIC & 0 & 6.127 & 10.917 \\
\hline
\end{tabular}

$\Lambda$ HDE model is smaller than that of the HDE model. Note that $C>1$ corresponds to a quintessence type HDE, while $C<1$ corresponds to a phantom type HDE $\mathrm{Li}$ (2004). Therefore, for the $\Lambda$ HDE model, the HDE component is closer to a phantom DE than that of the original HDE model. Moreover, from this table we see that, the results of $\Omega_{m 0}$ and $\Omega_{b 0}$ of these three DE models are very close. This means that, although these three DE models have very different DE components, they indeed have a similar total fractional dark energy density, i.e. $\Omega_{d e 0} \sim 0.7$. Moreover, to assess these three DE models, we list the $\chi_{\min }^{2} / \operatorname{dof}$, the Akaike information criteria (AIC) Akaike (1974) and the Bayesian information criteria (BIC) Schwarz (1978) of three DE models in the table 1 The AIC and the BIC are defined as:

$$
\mathrm{AIC}=\chi_{\min }^{2}+2 k, \mathrm{BIC}=\chi_{\min }^{2}+k \ln N
$$

where $k$ is the number of free parameters, and $N$ is the number of data points. From the table 1 , we find that, all the three criterias (i.e. $\chi_{\min }^{2} / d o f$, AIC and BIC) indicate that the $\Lambda \mathrm{CDM}$ model gives the best cosmology-fits among the three DE models. In other words, from the sight of model fitting, the $\Lambda \mathrm{CDM}$ model is the best DE model of three DE models. However, there are some other problems in the $\Lambda \mathrm{CDM}$ model, such as the cosmological coincidence problem. Moreover, this problem can be solved in the framework of the $\Lambda$ HDE model (see Appendix A. Therefore, it is necessary to consider the case of beyond the standard cosmological model.

Therefore, we can conclude that making use of the cosmology-fit results given by the current observations cannot distinguish the $\Lambda$ HDE model from the $\Lambda$ CDM and the HDE model. In other words, even if we can obtain the exact value of $\Omega_{d e 0}$, we still cannot determine the specific composition of DE. This indicates that, the possibility that DE may contain multiple components cannot be rule out by the cosmological observations.

\subsection{The impact of curvature on the cosmic evolutions and the cosmic fates of the $\Lambda$ HDE model}

In this subsection, we investigate the impact of curvature on the cosmic evolutions and the corresponding cosmic fates of the $\Lambda \mathrm{HDE}$ model from both the theoretical and the observational aspects. It should be mentioned that this topic has not been studied in the past.

Let us start from the theoretical side. From the Friedmann 
equation 2 we can derive

$$
\left(1-\Omega_{h d e}\right) H^{2}=\Omega_{m 0} H_{0}^{2} a^{-3}+\Omega_{r 0} H_{0}^{2} a^{-4}+\Omega_{k 0} H_{0}^{2} a^{-2}+\Omega_{\Lambda 0} H_{0}^{2},
$$

where $a$ is the scale factor. Defining a new function

$$
f(a) \equiv \Omega_{m 0} a^{-1}+\Omega_{r 0} a^{-2}+\Omega_{k 0}+\Omega_{\Lambda 0} a^{2},
$$

we can obtain (see Appendix B

$a \frac{d \Omega_{h d e}}{d a}=\left[\frac{2}{C} \sqrt{\Omega_{h d e}+C^{2} \Omega_{k}}-a \frac{d}{d a} \ln |f(a)|\right] \Omega_{h d e}\left(1-\Omega_{h d e}\right)$.

It has been proved that, for the $\Lambda$ HDE model, the scale factor $a$ can eventually approach infinity in a flat universe Hu et al. (2015). In this work, we prove that this conclusion can be extended to the case of a non-flat universe (see Appendix B). Then, when $a$ is large enough, the following approximation condition

$$
a \frac{d}{d a} \ln |f(a)|=\frac{2 \Omega_{\Lambda 0} a^{2}-\Omega_{m 0} a^{-1}-2 \Omega_{r 0} a^{-2}}{\Omega_{\Lambda 0} a^{2}+\Omega_{m 0} a^{-1}+\Omega_{r 0} a^{-2}+\Omega_{k 0}} \approx 2
$$

will be satisfied. In addition, for this case, the term of $\Omega_{k}$ can be also neglected. Therefore, we can obtain the following equation

$$
a \frac{d}{d a} \ln \left|\frac{\Omega_{h d e}}{1-\Omega_{h d e}}\right|=\frac{2}{C} \sqrt{\Omega_{h d e}}-2 .
$$

Notice that Eq. 30 has the same form with the case of a flat universe. This equation has an asymptotic solution

$$
\begin{aligned}
\ln a+x_{1}= & \frac{C}{2 C-2} \ln \left|1-\sqrt{\Omega_{h d e}}\right|-\frac{1}{2} \ln \Omega_{h d e} \\
& -\frac{1}{\left(C^{2}-1\right)} \ln \left|\sqrt{\Omega_{h d e}}-C\right| \\
& +\frac{C}{2 C+2} \ln \left(1+\sqrt{\Omega_{h d e}}\right),
\end{aligned}
$$

where $x_{1}$ is the constant of integration. This solution is also the same as the result obtained in a flat universe $\mathrm{Hu}$ et al. (2015). Therefore, we can conclude that the asymptotic solution of $\Omega_{h d e}$ obtained in a flat universe can be extended to the case of a non-flat universe.

By analyzing the properties of this analytical solution, in table 2 we list the evolution trends of $\Omega_{h d e}$ and the corresponding cosmic fates given by the various initial conditions. From this table we see that:

- If $C^{2}>1$, then for the $\Lambda$ HDE model, $\Omega_{h d e}$ will eventually approach 0 or $C^{2}$, and the universe will undergo an eternal accelerated expansion.

- If $C^{2}<1$ and $\Omega_{h d e}<C^{2}$, then for the $\Lambda$ HDE model, $\Omega_{h d e}$ will eventually approach 0 , and the universe will also undergo an eternal accelerated expansion.

- If $C^{2}<1$ and $\Omega_{h d e}>C^{2}$, then for the $\Lambda$ HDE model, $\Omega_{h d e}$ will eventually approach 1 , and the universe will finally encounter a big rip.

These results are also consistent with that obtained in a flat universe Hu et al. (2015).

From the above discussions, we can see that only performing a qualitative theoretical analysis is not enough to reflect the differences between a flat universe and a non-flat universe. For a further investigation, it is necessary to perform a quantitative numerical study. So in the Fig. 1, based on the cosmology-fit results given by the SNLS3+BAO1d+Planck 2015+GF data, we reconstruct the cosmic evolutions of $\Omega_{\Lambda}$ (left panel) and $\Omega_{h d e}$ (right panel) at $1 \sigma$ confidence region. It must be stressed that, this choice of redshift region is quite different from the case of Hu et al. (2015), in which the region $[-1,0]$ is not considered. This is because one of the main aims of the present work is to study the cosmic fate of the $\Lambda \mathrm{HDE}$ model, which has not been considered in $\mathrm{Hu}$ et al. (2015). From this figure, we can see that there is significant differences between a flat universe and a non-flat universe. For the flat case, when $z$ approaches $-1, \Omega_{\Lambda}$ may approach 0 or 1 (see the left panel); correspondingly, $\Omega_{h d e}$ may approach 1 or 0 (see the right panel). On the other hand, for the non-flat case, $\Omega_{\Lambda}$ will approach 0 (see the left panel); correspondingly, $\Omega_{h d e}$ will approach 1 (see the right panel). This means that, for this case the energy density of HDE will increase with time $t$, which is a typical feature of phantom DE. Therefore, compared with the case of a flat universe, considering curvature will make HDE closer to a phantom DE.

\subsection{The effects of adopting different types of observational data on the cosmic evolutions and the cosmic fates}

The discussions above only adopt the SNLS3+BAO1d+Planck $2015+\mathrm{GF}$ data. In this subsection, we explore the impact of adopting different types of observational data on the cosmic evolutions and the cosmic fates of $\Lambda \mathrm{HDE}$. It should be pointed out that this topic has not been studied in the previous literatures.

\subsubsection{Impacts of adopting different $S N$ data}

Firstly, we study the impact of adopting different SN datasets. For convenience, here we use "SNLS3" and "JLA" to represent the SNLS3+BAO1d+Planck 2015+GF and the JLA+BAO1d+Planck $2015+\mathrm{GF}$ datasets, respectively. Making use of these two SN datasets, in Fig. 2 we reconstruct the evolutions of $\Omega_{\Lambda}$ (left panel) and $\Omega_{h d e}$ (right panel) at $1 \sigma$ confidence region. From this figure we see that, the $1 \sigma$ region of $\Omega_{\Lambda}$ given by the "JLA" dataset has two possibilities: $\Omega_{\Lambda}$ may eventually approach 1 or 0 (see the left panel); correspondingly, $\Omega_{\text {hde }}$ may eventually approach 0 or 1 at $1 \sigma \mathrm{CL}$ (see the right panel). In contrast, the $1 \sigma$ region of $\Omega_{\Lambda}$ given by the "SNLS3" dataset has only one possibility: $\Omega_{\Lambda}$ will eventually approach 0 (see the left panel); correspondingly, $\Omega_{h d e}$ will eventually approach 1 at $1 \sigma$ CL (see the right panel). This means that the adopting "SNLS3" data will yield a HDE dominated universe at $1 \sigma \mathrm{CL}$. In other words, compared with JLA dataset, SNLS3 dataset more favors a phantom type HDE.

\subsubsection{Impacts of adopting different BAO data}

Next, we explore the effects of adopting different BAO data. For convenience, here we use "BAO1d" and "BAO2d" to represent the SNLS3+BAO1d+Planck 2015+GF and the SNLS3+BAO2d+Planck 2015+GF datasets, respectively. In Fig. 3. by using the "BAO1d" and the "BAO2d" datasets, we reconstruct the evolutions of $\Omega_{\Lambda}$ (left panel) and $\Omega_{h d e}$ (right panel) at $1 \sigma$ confidence region. It is clear that "BAO1d" dataset, which is indeed the same as the "SNLS3" dataset, favors a HDE dominated universe at $1 \sigma \mathrm{CL}$. In addition, the evolutionary trajectory of $\Omega_{\Lambda}$ and $\Omega_{h d e}$ given by the "BAO2d" dataset almost overlap with the results of "BAO1d" dataset. this means that using different BAO data have little impact on the cosmic evolutions of the $\Lambda$ HDE model. 

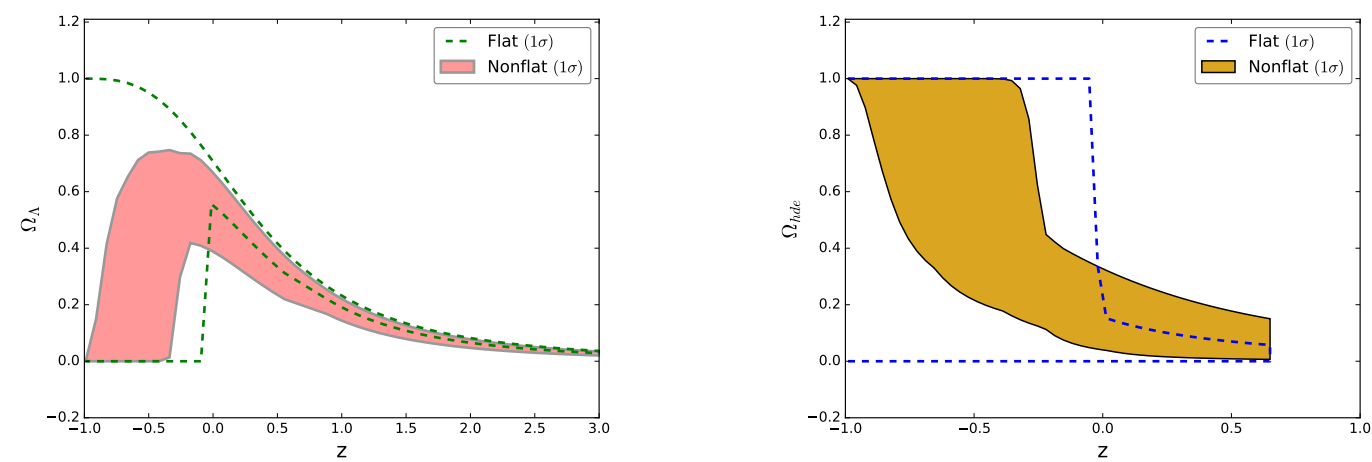

Figure 1. The cosmic evolutions of $\Omega_{\Lambda}$ (left panel) and $\Omega_{h d e}$ (right panel). In the left panel, the region inside green lines and the pink filled region denote the $1 \sigma$ regions of $\Omega_{\Lambda}$ in a flat universe and a non-flat universe, respectively; In the right panel, the region inside blue lines and the gold filled region denote the $1 \sigma$ regions of $\Omega_{h d e}$ in a flat universe and a non-flat universe, respectively.
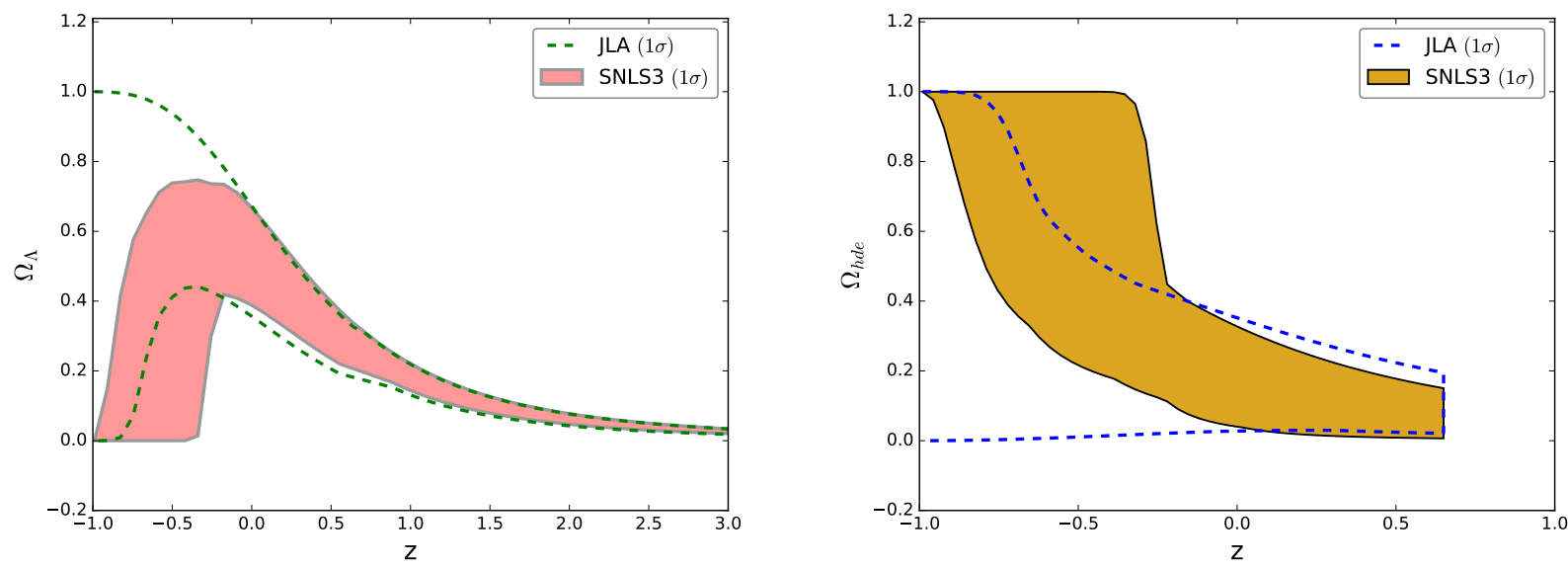

Figure 2. The cosmic evolutions of $\Omega_{\Lambda}$ (left panel) and $\Omega_{h d e}$ (right panel). In the left panel, the region inside green lines and the pink filled region denote the $1 \sigma$ regions of $\Omega_{\Lambda}$ given by the JLA data and the SNLS3 data, respectively; In the right panel, the region inside blue lines and the gold filled region denote the $1 \sigma$ regions of $\Omega_{h d e}$ given by the JLA data and the SNLS3 data, respectively. "JLA" and"SNLS3" denote the JLA+BAO1d+Planck 2015+GF data and the SNLS3+BAO1d+Planck 2015+GF data, respectively.
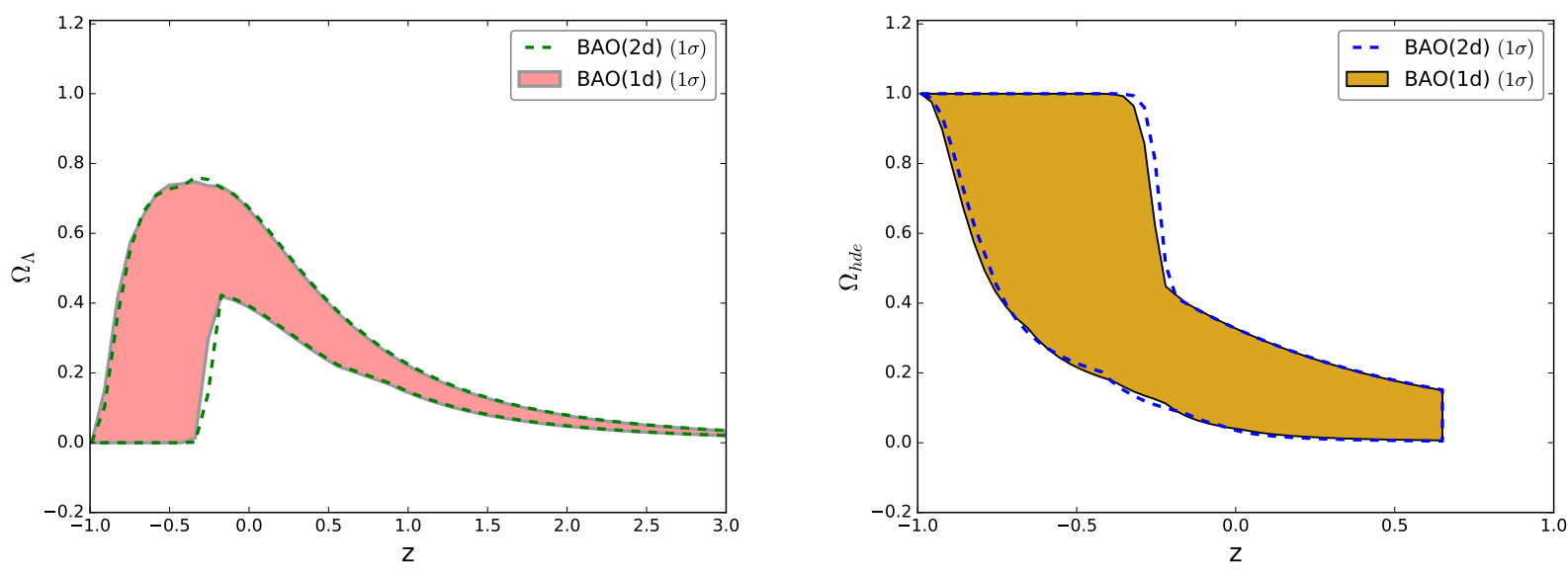

Figure 3. The cosmic evolutions of $\Omega_{\Lambda}$ (left panel) and $\Omega_{h d e}$ (right panel). In the left panel, the region inside green lines and the pink filled region denote the $1 \sigma$ regions of $\Omega_{\Lambda}$ given by the BAO2d data and the BAO1d data, respectively; In the right panel, the region inside blue lines and the gold filled region denote the $1 \sigma$ regions of $\Omega_{h d e}$ given by the BAO2d data and the BAO1d data, respectively. "BAO1d" and "BAO2d" denote the SNLS3+BAO1d+Planck 2015+GF data and the SNLS3+BAO2d+Planck 2015+GF data, respectively. 
Table 2. The finally evolution trend of $\Omega_{h d e}$ and the corresponding cosmic fate for different initial conditions. The initial conditions are listed in the first column. The corresponding evolution trends of $\Omega_{h d e}$ are given in the second column. The final fates of the universe are presented in the last column.

\begin{tabular}{ccc}
\hline \hline Initial condition & evolution trend & fate of universe \\
\hline$\Omega_{h d e}<1<C^{2}$ & $\Omega_{h d e}$ will decrease to 0 & eternal accelerated expansion \\
$\Omega_{h d e}<C^{2}<1$ & $\Omega_{h d e}$ will decrease to 0 & eternal accelerated expansion \\
$C^{2}<\Omega_{h d e}<1$ & $\Omega_{h d e}$ will increase to 1 & big rip \\
$C^{2}<1<\Omega_{h d e}$ & $\Omega_{h d e}$ will decrease to 1 & big rip \\
$1<\Omega_{h d e}<C^{2}$ & $\Omega_{h d e}$ will increase to $C^{2}$ & eternal accelerated expansion \\
$1<C^{2}<\Omega_{h d e}$ & $\Omega_{h d e}$ will decrease to $C^{2}$ & eternal accelerated expansion \\
\hline
\end{tabular}
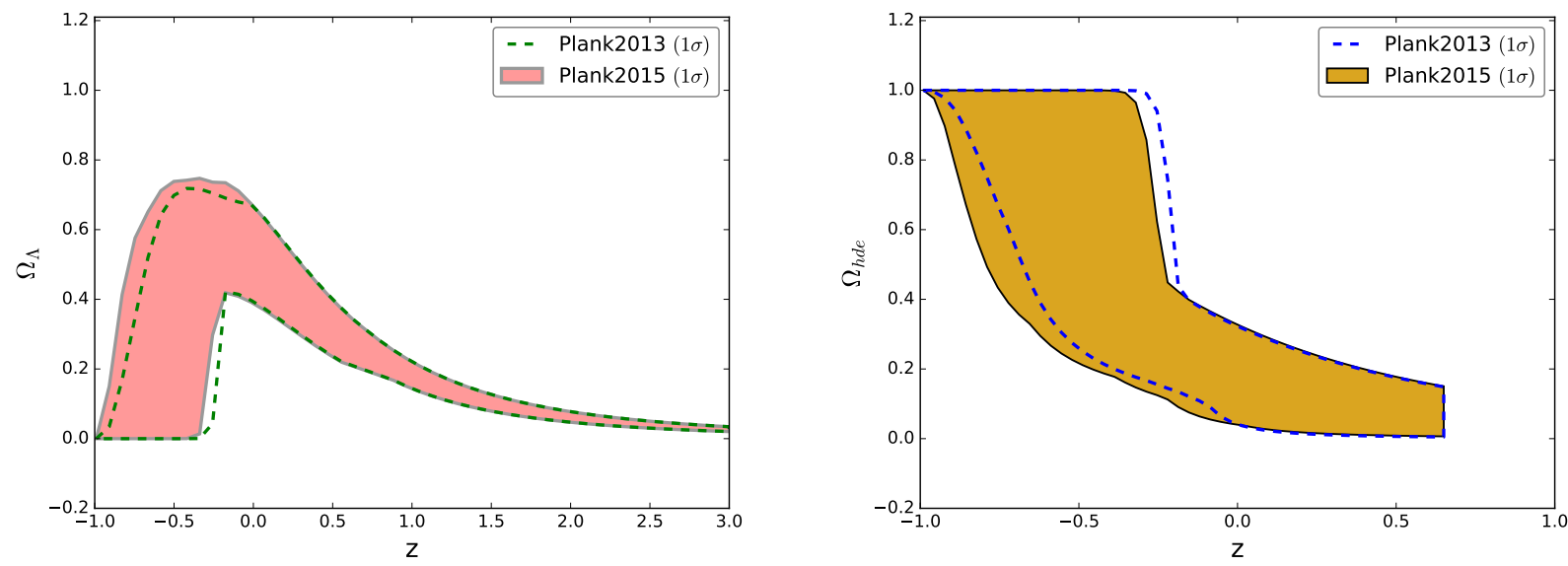

Figure 4. The cosmic evolutions of $\Omega_{\Lambda}$ (left panel) and $\Omega_{h d e}$ (right panel). In the left panel, the region inside green lines and the pink filled region denote the $1 \sigma$ regions of $\Omega_{\Lambda}$ given by the Planck 2013 data and the Planck 2015 data, respectively; In the right panel, the region inside blue lines and the gold filled region denote the $1 \sigma$ regions of $\Omega_{h d e}$ given by the Planck 2013 data and the Planck 2015 data, respectively. "Planck 2013" and "Planck 2015" denote the SNLS3+BAO1d+Planck 2013+GF data and the SNLS3+BAO1d+Planck 2015+GF data, respectively.

\subsubsection{Impacts of adopting different CMB data}

Then, we study the effects of adopting different CMB data. Here we use "Planck 2015" and "Planck 2013" to represent the SNLS3+BAO1d+Planck 2015+GF and the SNLS3+BAO1d+Planck 2013+GF datasets, respectively. In Fig. 4. by adopting these two datasets, we reconstruct the evolutions of $\Omega_{\Lambda}$ (left panel) and $\Omega_{h d e}$ (right panel) at $1 \sigma$ confidence region. Again, the "Planck 2015" dataset, which is indeed the same as the "SNLS3" dataset, favors a HDE dominated universe at $1 \sigma$ CL. In addition, the evolutionary trajectories of $\Omega_{\Lambda}$ and $\Omega_{h d e}$ given by the "Planck 2013" dataset almost overlap with the results of "Planck 2015" dataset. this result is just the same as the case of using different BAO data.

\subsubsection{Impacts of adding growth factor data or not}

Finally, we discuss the impact of adding GF data or not. Here we use "With GF" and "Without GF" to represent the SNLS3+BAO1d+Planck 2015+GF and the SNLS3+BAO1d+Planck 2015 datasets, respectively. In Fig. 5 by making use of these two datasets, we reconstruct the evolutions of $\Omega_{\Lambda}$ (left panel) and $\Omega_{h d e}$ (right panel) at $1 \sigma$ confidence region. From this figure we see that, although there are small differences between the evolutionary trajectories given by the "Without GF" and the "With GF" datasets, the overall evolutionary trends of the "Without GF" and the "With GF" cases are exactly the same.
Therefore, we can conclude that adding the GF data or not will not have any significant impact on the cosmic evolutions of the $\Lambda \mathrm{HDE}$ model.

\section{SUMMARY}

In the previous theoretical studies of DE, DE is always viewed as a single component. So far as we know, the $\Lambda$ HDE model, which is proposed in $\mathrm{Hu}$ et al. (2015), is the first theoretical attempt to explore the possibility that DE contains multiple components. In Hu et al. (2015), we have performed some simply analyses on this model. However, there are still some shortcomings for Ref. Hu et al. I (2015): (1) the cosmology-fit results of the $\Lambda$ HDE model have not been compared with the results of other DE models; (2) the impact of curvature on the cosmic evolutions and the cosmic fates of the $\Lambda$ HDE model have not been considered; (3) the effects of adopting different types of observational data have not been taken into account.

The main aim of the present work is to give a more comprehensive and systematic investigation on the cosmological implications of the $\Lambda \mathrm{HDE}$ model. In particular, by combining the qualitative theoretical analyses with the quantitative numerical studies, we explore in details the issues mentioned above. It should be mentioned that, different from Ref. Hu et al. (2015), in this work we study a new version of $\Lambda \mathrm{HDE}$ model: in the deceleration expansion stage, DE only contains the CC term; in the accelerated expansion 

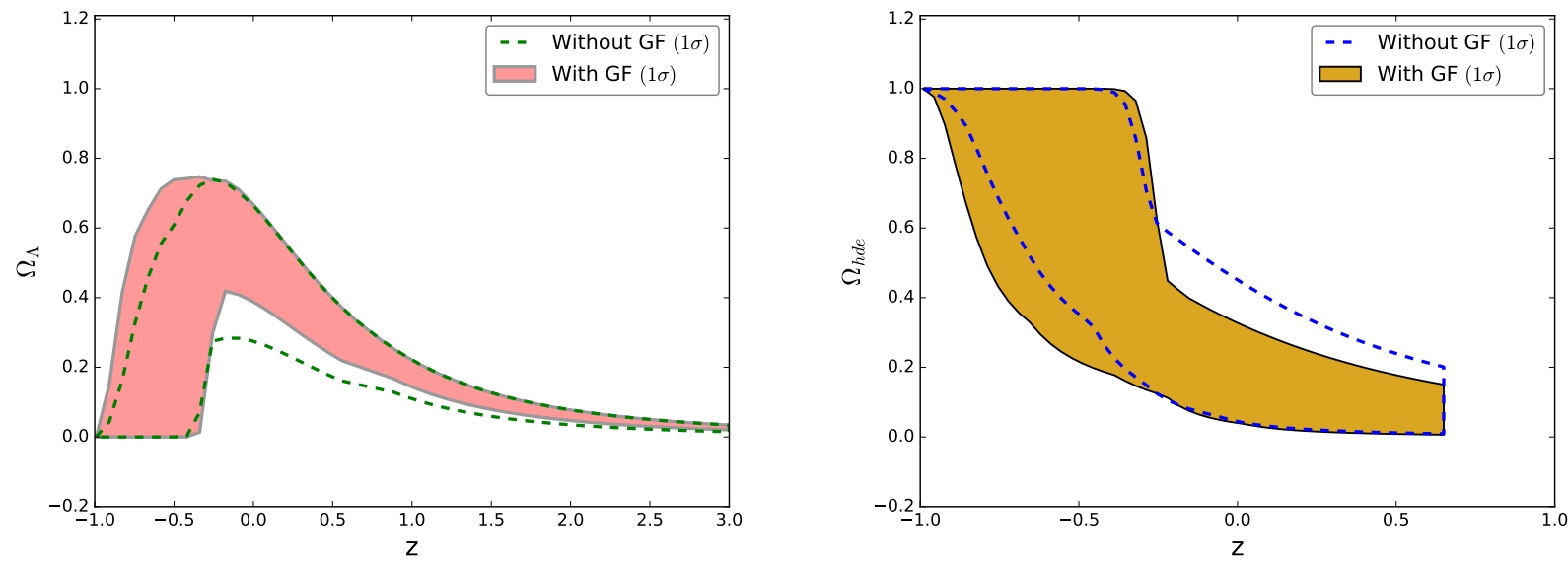

Figure 5. The cosmic evolutions of $\Omega_{\Lambda}$ (left panel) and $\Omega_{h d e}$ (right panel). In the left panel, the region inside green lines and the pink filled region denote the $1 \sigma$ regions of $\Omega_{\Lambda}$ for the cases without and with GF data, respectively; In the right panel, the region inside blue lines and the gold filled region denote the $1 \sigma$ regions of $\Omega_{h d e}$ for the cases without and with GF data, respectively. "Without GF" and "With GF" denote the SNLS3+BAO1d+Planck 2015 data and the SNLS3+BAO1d+Planck 2015+GF data, respectively.

stage, DE contains both the CC and the HDE. In addition, the observational data used in this work includes two types of SN data (SNLS3 and JLA), two types of BAO data (BAO1d and BAO2d), two types of CMB data (Planck 2015 and Planck 2013) and the GF data.

Our conclusions are as follows:

- The $\Lambda$ HDE model has a similar value of $\Omega_{d e 0}$ with the $\Lambda$ CDM and the HDE model(see Table 1); this implies that making use of the current observations cannot distinguish the $\Lambda \mathrm{HDE}$ model from the other two models. In other words, even if we can obtain the exact value of $\Omega_{d e 0}$, we still cannot determine the specific composition of DE. This indicates that, the possibility that DE may contain multiple components cannot be rule out by the cosmological observations.

- The qualitative theoretical analysis shows that, for the $\Lambda \mathrm{HDE}$ model, the asymptotic solution of $\Omega_{h d e}$ and the corresponding cosmic fate (see Table 2) in a flat universe can be extended to the case of a non-flat universe. On the other side, the quantitative numerical study shows that, considering curvature will make HDE closer to a phantom dark energy. (see Fig. 1). For this case, the universe will finally encounter a big rip

- compared with JLA dataset, SNLS3 dataset more favors a phantom type HDE (see Fig. 2). In contrast, using other types of observational data have no significant impact on the cosmic evolutions of $\Omega_{\Lambda}$ and $\Omega_{h d e}$ (see Fig. 3 . Fig. 4 and Fig. 5). These observational data all favor a phantom type HDE, showing that the universe will finally encounter a big rip.

There are still many other topics, such as perturbation Li, Lin \& Wang (2008), interaction Li et al. (2009b), neutrino Li et al. (2013), cosmic age Lan et al. (2010); Wang, Li \& Li (2010), standard siren Wang et al. (2008); Wang (2010) and so on, deserve to be studied in the framework of the $\Lambda \mathrm{HDE}$ model. In addition, it is also interesting to make use of various diagnosis tools to analyze the cosmic evolution of the $\Lambda$ HDE model Zhou \& Wang (2016). These will be done in future work.

\section{ACKNOWLEDGMENTS}

We thank the referee for very helpful suggestions. SW is supported by the National Natural Science Foundation of China under Grant No. 11405024 and the Fundamental Research Funds for the Central Universities under Grant No. 16lgpy50. ML is supported by the National Natural Science Foundation of China (Grant No. 11275247, and Grant No. 11335012) and a 985 grant at Sun Yat-Sen University.

\section{APPENDIX A: THE SOLUTION OF COSMOLOGICAL COINCIDENCE PROBLEM IN THE FRAMEWORK OF THE $\Lambda$ HDE MODEL}

The cosmological coincidence problem was first proposed in a conference Steinhardt (1997). This problem can be expressed a problem of why the radiation energy density is far greater than the dark energy density at the onset of the radiation dominated epoch. Now, let us discuss the possible solution to the cosmological coincidence problem in the framework of the $\Lambda$ HDE model. Since the future event horizon does exist in the inflation stage, the HDE may also exist in this stage; we can assume that, in the standard slow-roll inflation epoch there are two energy components: the HDE and the inflation energy. If the latter is almost constant, we can derive the evolution equation of $\Omega_{h d e}$ :

$$
a \frac{d}{d a} \ln \left|\frac{\Omega_{h d e}}{1-\Omega_{h d e}}\right|=\frac{2}{C} \sqrt{\Omega_{h d e}}-2 .
$$

By solving the above equation, we find that, when the scale factor $a$ is large enough,

$$
\Omega_{h d e} \sim a^{-2} .
$$

From the above equation, we can see that, in the end of inflation, $\Omega_{h d e}$ will approach a small number. Meanwhile, we assume that most of the inflation energy is decayed into radiation and the other part of the inflation energy is included in a CC. Therefore, if the number of e-folds is reasonable, we can get a very tiny ratio between the dark energy density and the radiation density at the onset 
of the radiation dominated epoch. This means that, the inflation can naturally solve the cosmological coincidence problem without any fine-tuned initial conditions.

\section{APPENDIX B: A PROOF THAT SCALE FACTOR $A$ HAS NO MAXIMUM IN THE $\Lambda$ HDE MODEL}

In this appendix, we prove that, in the framework of the $\Lambda \mathrm{HDE}$ model, the scale factor $a$ does not have a maximum.

Firstly, we will proof that, if $a$ is finite, $\rho_{h d e}=3 C^{2} M_{p}^{2} / L^{2} \neq$ 0 is always satisfied. We notice that the cutoff scale length $L$ can be written:

$$
L=\operatorname{ar}(t)
$$

where $r(t)$ is defined as

$$
r(t)=\frac{1}{\sqrt{\left|\Omega_{k 0}\right|} H_{0}} \operatorname{sinn}\left(\sqrt{\left|\Omega_{k 0}\right|} H_{0} \int_{t}^{+\infty} \frac{d t^{\prime}}{a}\right) .
$$

The evolution of $r(t)$ has two possibilities:

- If $\Omega_{k 0}<0$, then $r(t)$ must be a bounded function, because sinn takes a $\sin$ function form.

- If $\Omega_{k 0} \geqslant 0$, then $r(t)$ must be a decreasing function. For the $\Lambda$ HDE model, the current HDE density $\rho_{h d e 0}>0$ is always satisfied, then $r\left(t_{0}\right)$ must be finite. So in the future, $r(t)<r\left(t_{0}\right)$ is always satisfied.

The discussions above imply that $r(t)$ must be finite, which is independent of the specific value of $\Omega_{k 0}$. Therefore, when $a$ is finite, $r$ is also finite, and $\rho_{h d e}=3 C^{2} M_{p}^{2} /(a r)^{2} \neq 0$.

Secondly, we will proof that $a$ does not have a maximum. Assuming that $a$ has a maximum $a_{m}$, then $\left.H\right|_{a=a_{m}}=\left.\frac{\dot{a}}{a}\right|_{a=a_{m}}=0$. Notice that $\left.\rho_{h d e}\right|_{a=a_{m}} \neq 0$, so

$$
\left.\Omega_{h d e}\right|_{a=a_{m}}=\left.\frac{\rho_{h d e}}{3 M_{p l}^{2} H^{2}}\right|_{a=a_{m}} \rightarrow \infty .
$$

In the following, we will prove that the Eq. B3 contradict with the Friedmann equation, which has the form

$$
3 M_{p l}^{2} H^{2}=\rho_{m}+\rho_{r}+\rho_{k}+\rho_{\Lambda}+\rho_{h d e} .
$$

Let us define that

$$
\begin{aligned}
f(a) & \equiv \Omega_{m 0} a^{-1}+\Omega_{r 0} a^{-2}+\Omega_{k 0}+\Omega_{\Lambda 0} a^{2}, \\
g(a) & \equiv a \frac{d}{d a} \ln |f(a)| .
\end{aligned}
$$

It is easily to get

$$
g(a)=\frac{2 \Omega_{\Lambda 0} a^{2}-\Omega_{m 0} a^{-1}-2 \Omega_{r 0} a^{-2}}{f(a)} .
$$

Moreover, from the Friedmann equation B4 we can derive

$$
a \frac{d \Omega_{h d e}}{d a}=\left[\frac{2}{C} \sqrt{\Omega_{h d e}+C^{2} \Omega_{k}}-g(a)\right] \Omega_{h d e}\left(1-\Omega_{h d e}\right) .
$$

Due to that the evolution of $\Omega_{h d e}$ mainly depends on the terms in the right hand of Eq. B8 we will discuss the values of these terms in the following.

Firstly, let's consider the function $g(a)$. From the Eq. B4 we get $\left.\left(\rho_{m}+\rho_{r}+\rho_{k}+\rho_{\Lambda}\right)\right|_{a=a_{m}}=-\left.\rho_{\text {hde }}\right|_{a=a_{m}} \neq 0$. Then,

$$
\left.f\left(a_{m}\right) \propto\left(\rho_{m}+\rho_{r}+\rho_{k}+\rho_{\Lambda}\right)\right|_{a=a_{m}} \neq 0 .
$$

Based on the Eq. B7 we can see that $g\left(a_{m}\right)$ must be finite.

Then, let us discuss other terms in Eq. B8 When $a$ approaches $a_{m}$,

(C) 0000 RAS, MNRAS 000, 000-000
- if $\Omega_{k}>0$, then $\sqrt{\Omega_{h d e}+C^{2} \Omega_{k}}$ will approach infinity apparently;

- if $\Omega_{k}<0$, notice that the EoS of HDE satisfies Hu et al. (2015) $w_{h d e}=-\frac{1}{3}-\frac{2}{3} \sqrt{\frac{\Omega_{h d e}}{C^{2}}+\Omega_{k}}<-\frac{1}{3}$, we can get

$$
\begin{aligned}
\sqrt{\Omega_{h d e}+C^{2} \Omega_{k}} & =\sqrt{\Omega_{h d e}\left(1+C^{2} \frac{\rho_{k 0}}{\rho_{h d e 0}} a^{\left.1+3 w_{h d e}\right)}\right.} \\
& >\sqrt{\Omega_{h d e}\left(1+C^{2} \frac{\rho_{k 0}}{\rho_{h d e 0}}\right)} .
\end{aligned}
$$

Since $\sqrt{\Omega_{h d e}\left(1+C^{2} \frac{\rho_{k 0}}{\rho_{h d e 0}}\right)}$ will approach infinity when $a \rightarrow$ $a_{m}, \sqrt{\Omega_{h d e}+C^{2} \Omega_{k}}$ will still approach infinity.

Therefore, the relation

$$
\left.\sqrt{\Omega_{h d e}+C^{2} \Omega_{k}}\right|_{a=a_{m}} \rightarrow \infty
$$

is also independent of the specific value of $\Omega_{k 0}$.

Finally, let's discuss the evolution of $\Omega_{h d e}$. When $a$ approaches $a_{m}, \sqrt{\Omega_{h d e}+C^{2} \Omega_{k}}>\frac{C}{2} g(a)$ and $\Omega_{h d e}>1$ will always be satisfied. So according to the Eq. B8, we can get

$$
\frac{d}{d a} \sqrt{\Omega_{h d e}}<0
$$

It is clear that $\Omega_{h d e}$ is a decreasing function of $a$, and this means that $\Omega_{h d e}$ will never approach infinity in the future. This result apparently contradicts with the Eq. B3 So the previous assumption about $a$ is wrong, and $a$ has no maximum.

\section{REFERENCES}

Abazajian K. N. et al., 2009, ApJS, 182, 543

Ade P. A. R. et al., 2015a, arXiv:1502.01589

Ade P. A. R. et al., 2015b, arXiv:1502.01590

Akaike H., 1974, IEEE Trans. Automatic Control, 19, 716.

Armendariz-Picon C., Damour T., Mukhanov V., 1999, Phys. Lett. B, 458, 209

Bengochea G. R., 2011, Phys. Lett. B 696, 5

Bengochea G. R., \& De Rossi, M. E., 2014, Phys. Lett. B 733, 258

Bento M. C., Bertolami O., Sen A. A., 2002, Phys. Rev. D 66, 043507

Bertone G., Hooper D., Silk J., 2005, Phys. Rept. 405, 279

Betoule M. et al. 2014, Astron. Astrophys. 568, A22

Beutler F. et al., 2011, MNRAS, 416, 3017

Beutler F. et al., 2012, MNRAS, 423, 3430

Blake C. et al., 2011, MNRAS, 415, 2876.

Caldwell R. R., 2002, Phys. Lett. B 545, 23

Caldwell R. R., and Kamionkowski M., 2009, Ann. Rev. Nucl. Part. Sci. 59, 397

Cai R. G., 2007, Phys. Lett. B 657, 228

Chang Z., Wu F. Q., Zhang X., 2006, Phys. Lett. B 633, 14-18

Conley A. et al., 2011, Astrophys. J. Suppl. 192, 1

Eisenstein D. J. et al., 2011, AJ, 142, 72

Freese K., Adams F. C., Frieman J. A. et al. 1987, Nucl. Phys. B 287, 797-814

Frieman J., Turner M., Huterer D., 2008, Ann. Rev. Astron. Astrophys 46,385

Gao C. J., Chen X. L., Shen Y. G., 2009, Phys. Rev. D 79, 043511

Guzzo L. et al., 2008, Nature, 451, 541

't Hooft G., 1993, arXiv:gr-qc/9310026. 
Hawkins C. et al., 2003, MNRAS, 346, 78

Hemantha M. D. P., Wang Y., Chuang C. H., 2014, MNRAS, 445, 3737

Horvat R., 2004, Phys.Rev.D, 70, 087301

Hu W., Sugiyama N., 1996, ApJ, 471 : 542-470

Hu Y., Li M., Li N. et al., 2015, JCAP 1508, 012

Hu Y., Li M., Li N. et al., 2016, Astron. Astrophys. 592, A101

Huang Q. G., Gong Y. G., 2004, JCAP 0408, 006

Huang Q. G., Li M., 2004, JCAP 0408, 013

Huang Q. G., Li M., Li X. D. et al., 2009, Phys. Rev. D 80, 083515

Huang Q. G., Wang S., Wang K., 2015, JCAP 1512, 022

Kamenshchik A. Y., Moschella U., Pasquier V., 2001, Phys. Lett. B, 511, 265

Lan M. X., Li M., Li X. D. et al., 2010, Phys. Rev. D 82, 023516

Li M., 2004, Phys. Lett. B 603, 1.

Li M., Li N., Wang S. et al., 2016, Mon. Not. Roy. Astron. Soc. 460, 2586

Li M., Li X. D., Ma Y. Z. et al., 2013, JCAP 1309, 021

Li M., Li X. D., Meng J. et al. 2013, Phys. Rev. D 88, 023503

Li M., Li X. D., Wang S. et al., 2011, Commun. Theor. Phys. 56, 525

Li M., Li X. D., Wang S. et al., 2013, Frontiers of Physics 8, 828

Li M., Li X. D., Wang S. et al., 2009b, JCAP 0912014

Li M., Li X. D., Wang S. et al., 2009a, JCAP 0906, 036

Li M., Lin C. S., Wang Y., 2008, JCAP 0805, 023

Li M. \& Miao R. X., 2012, arXiv:1210.0966

Li M., Miao R. X., Pang Y., 2010, Phys.Lett.B, 689, 55

Li M. \& Wang Y., 2010, Phys. Lett. B 687, 243

Li X. D., Li S., Wang S. et al., 2011, JCAP 1107, 011

Li X. D., Wang S., Huang Q. G. et al., 2012, Sci. China Phys. Mech. Astron. G 55, 1330

Li Y. H., Wang S., Li X. D. et al., 2013, JCAP 1302, 033

Malquarti M., Copeland E. J, Liddle A. R et al. 2003, Phys. Rev. D 67, 123503

Nojiri S., Odintsov S. D., 2006, Gen. Rel. Grav. 38, 1285

Pavlov A., Farooq O., Ratra B., 2014, Phys. Rev. D 90, 023006

Pavon D., Zimdahl W., 2005, Phys. Lett. B 628, 206-210

Perlmutter S. et al., 1999, ApJ, 517, 565

Polyakov A. M., 2010, Nuclear Phys. B834, 316

Riess A. G., et al. 1998, AJ, 116, 1009

Ross N. P. et al., 2007, MNRAS, 381, 573

Saridakis E. N., 2008, Phys. Lett. B 661, 335

Schwarz G., 1978, Annals of Statistics, 6461

Setare M. R., 2006, Phys. Lett. B 644, 99

Setare M. R., 2007, Phys. Lett. B 653, 116

Shen J. Y., Wang B., Abdalla E. et al., 2005, Phys. Lett. B 609, 200

Song Y. S., Percival W. J., 2009, J. Cosmology Astropart. Phys., 0910, 004

Steinhardt P. J., 1997, Critical Problems in Physics, Princeton University Press, Princeton, NJ

Steinhardt P. J., Wang L. M., Zlatev I., 1999, Phys. Rev. D, 59, 123504.

Susskind L., 1995, J. Math. Phys. 36, 6377

Torre A. et al., 2013, arXiv:1303.2622

Wang B., Gong Y. G., Abdalla E., 2005, Phys. Lett. B 624, 141

Wang S., 2010, Phys. Rev. D 81, 023002

Wang S., Geng J. J., Hu Y. L. et al., 2015, Sci. China Phys. Mech. Astron. 58: 019801.

Wang S., Hu Y., Li M., 2015, arXiv:1506.08274 Sci. China Phys. Mech. Astron in press

Wang S., Hu Y., Li M. et al., 2016, ApJ 821, 60
Wang S., Li X. D., Li M., 2010, Phys. Rev. D 82, 103006

Wang S., Li X. D., Li M., 2011, Phys. Rev. D 83, 023010

Wang S., Li Y. H., Zhang X., 2014, Phys. Rev. D 89, 063524.

Wang S., Wang Y., 2013a, Phys. Rev. D 88, 043511.

Wang S., Wang Y., Li M., 2016, arXiv:1612.00345

Wang S., Wang Y. Z., Geng J. J. et al., 2014, Eur. Phys. J. C 74, 3148.

Wang S., Wang Y. Z., Zhang X., 2014, Commun. Theor. Phys. 62, 927.

Wang S., Wen S., Li M., 2016, arXiv:1606.01779

Wang S., Zhang Y., 2008, Phys. Lett. B 669, 201

Wang S., Zhang Y., Xia T.Y., 2008, JCAP 0810, 037

Wang S., Zhang Y., Xia T.Y. et al. 2008, Phys. Rev. D 77, 104016

Wang Y., 2014, MNRAS, 443, 2950

Wang Y., Dai M. 2016 Phys. Rev. D 94, 083521

Wang Y., Wang, S. 2013b, Phys. Rev. D, 88, 043522

Wei H. \& Cai R. G., Eur. Phys. J. C 59, 99

Wei H., Cai R. G., Zeng D. F., 2005, Class. Quant. Grav. 22, 3189

Witten E., 2000, Lecture given at Conference: C00-02-23, p.27-36

Proceedings

Zhang W. S., Cheng C., Huang Q. G., et al., 2012, Sci. China Phys. Mech. Astron. 55: 2244

Zhang X. \& Wu F. Q., 2007, Phys. Rev. D 76, 023502

Zhang Y., Xia T. Y., Zhao W., 2007, Class. Quant. Grav. 24, 3309

Zhang Z. H., Li M., Li X. D. et al., 2012, Mod. Phys. Lett. A27 1250115

Zhou L. J. \& Wang S., 2016, Sci. China Phys. Mech. Astron. 59, 670411

Zlatev I., Wang L. M., Steinhardt P.J., 1999, Phys. Rev. Lett. 82, 896 